\title{
Prevalence of sarcopenia in the world: a systematic review and meta- analysis of general population studies
}

\author{
Gita Shafiee ${ }^{1}$, Abbasali Keshtkar ${ }^{2}$, Akbar Soltani $^{3}$, Zeinab Ahadi ${ }^{1}$, Bagher Larijani ${ }^{3}$ and Ramin Heshmat ${ }^{14^{*}}$
}

\begin{abstract}
Background: Sarcopenia, an age-related decline in muscle mass and function, is one of the most important health problems in elderly with a high rate of adverse outcomes. However, several studies have investigated the prevalence of sarcopenia in the world, the results have been inconsistent. The current systematic review and meta- analysis study was conducted to estimate the overall prevalence of sarcopenia in both genders in different regions of the world.

Methods: Electronic databases, including MEDLINE (via PubMed), SCOPUS and Web of Science were searched between January 2009 and December 2016. The population- based studies that reported the prevalence of sarcopenia in healthy adults aged $\geq 60$ years using the European Working Group on Sarcopenia in Older People (EWGSOP), the International Working Group on Sarcopenia (IWGS) and Asian Working Group for Sarcopenia (AWGS) definitions, were selected. According to these consensual definitions, sarcopenia was defined by presence of low muscle mass (adjusted appendicular muscle mass for height) and muscle strength (handgrip strength) or physical performance (the usual gait speed).

The random effect model was used for estimation the prevalence of sarcopenia. The sex-specific prevalence of sarcopenia and 95\% confidence interval (Cl) were calculated using the Binomial Exact Method. Heterogeneity was assessed by subgroup analysis.
\end{abstract}

Results: Thirty- five articles met our inclusion criteria, with a total of 58404 individuals. The overall estimates of prevalence was 10\% (95\% Cl: 8-12\%) in men and 10\% (95\% Cl: 8-13\%) in women, respectively. The prevalence was higher among non- Asian than Asian individuals in both genders especially, when the Bio-electrical Impedance Analysis (BIA) was used to measure muscle mass (19\% vs $10 \%$ in men; $20 \%$ vs $11 \%$ in women).

Conclusion: Despite the differences encountered between the studies, regarding diagnostic tools used to measure of muscle mass and different regions of the world for estimating parameters of sarcopenia, present systematic review revealed that a substantial proportion of the old people has sarcopenia, even in healthy populations. However, sarcopenia is as a consequence of the aging progress, early diagnosis can prevent some adverse outcomes.

Keywords: Sarcopenia, Prevalence, General population, Systematic review

\footnotetext{
* Correspondence: rheshmat@tums.ac.ir

${ }^{1}$ Chronic Diseases Research Center, Endocrinology and Metabolism

Population Sciences Institute, Tehran University of Medical Sciences, Tehran, Iran

${ }^{4}$ Endocrinology and Metabolism Research Center (EMRC), Dr Shariati Hospital,

North Karegar St, Tehran 14114, Iran

Full list of author information is available at the end of the article
} 


\section{Background}

Sarcopenia is an age-related disease described by a progressive loss of muscle mass and function [1]. In addition, sarcopenia is a major clinical problem in public health of older people; with some adverse outcomes such as disability, poor quality of life, and increased risk of death [2-4]. The prevalence of sarcopenia is rising, which is as a result of population aging all over the world. Characteristics of the studied population (such as age, sex, race and differences in body composition in ethnic groups) and the methodology used to assess parameters of sarcopenia cause a wide variation in the rate of this disease [5, 6].

Researches in Europe and Asia have developed the consensus sets of definition and diagnostics criteria for sarcopenia based on its parameters. In 2010, a practical clinical definition was reported by the European Working Group on Sarcopenia in Older People (EWGSOP) [7]. The similar approaches for definition of sarcopenia were taken by the International Working Group on Sarcopenia (IWGS) [8] and Asian Working Group for Sarcopenia (AWGS) [9]. According to these consensual definitions, sarcopenia is defined by presence of low whole body or appendicular skeletal muscle mass in combination with poor muscle function.

There are some different methods used to define cutoff points for muscle mass and other parameters of sarcopenia. Although, the EWGSOP and the AWGS suggested cut-off values for muscle mass and function, the practice of young adult from the same population under study to determine these values is recommended. Hereof, researchers have shown significant differences and bias in the prevalence of Sarcopenia when different cut points of the distinct populations used instead of the original sex- specific group of subjects $[10,11]$.

Therefore, the use of different measurement tools, cutoff points and definitions may lead to different prevalence of sarcopenia and the results may be difficult to interpret. This may have important consequences on clinical researches and development of preventive and therapeutic strategies. Therefore, the European and Asian working groups recommended developing the systematic reviews of some aspects of sarcopenia. In this regard, we performed this study, aiming to systematically review the finding of all available studies in this issue and estimate the prevalence of sarcopenia using definitions as proposed by the EWGSOP and the AWGS; and also to assess potential sources of heterogeneity.

\section{Methods}

\section{Search strategy}

The search was carried out in three electronic databases, MEDLINE (via PubMed), SCOPUS and Web of Science between January 2009 and December 2016.
The pre-defined search terms were: "sarcopenia" or "muscle mass" and "aging" or "older people" or "elderly" and "prevalence" or "epidemiology" or "frequency" or "incidence". The list of references of articles was also reviewed for any additional papers. Furthermore, specialized journals and textbooks that were related to the topic were reviewed to gather data on sarcopenia prevalence. In addition, gray literatures, such as reports and conference presentations, were considered using the Google search engine to further ensure that pertinent articles were not missed. The reference lists of included studies were screened as well. Search was not limited by language.

\section{Study selection}

We included only studies that had enrolled communitydwelling participants aged 60 years and older among the normal population and population base approach sampling studies. Studies that prevalence of sarcopenia had been assessed according to the EWGSOP, AWGS or IWGS definitions of sarcopenia, i.e. based on muscle mass (adjusted appendicular muscle mass for height) and muscle strength (handgrip strength) or physical performance (the usual gait speed), were included. They were excluded if they only used muscle mass to define sarcopenia, and studies that were from clinical/hospital or care setting. Also, studies that were not published as full report like conferences abstract and letters to editors, case-report studies were excluded.

Research papers were selected based on titles and abstracts. The full texts of all selected publications were assessed for pertinence. If the full texts of the papers were not available, they were obtained through correspondence with the authors. The process initially performed by two investigators. A third investigator adjudicated discrepancies between the two reviewers.

\section{Data extraction and quality evaluation}

The methodological quality of the eligible articles was assessed using a modified STROBE checklist [12]. Two reviewers evaluated each article's quality based on the checklist, and if a consensus was not reached, a third reviewer reassessed the manuscript. We extracted from the articles the study characteristics (e.g. publication year, country of data collection), characteristics of target population (e.g. age, gender and sample size), assessment method used for each parameter (muscle mass, muscle strength, and muscle performance), cut-off values of each parameter, and sarcopenia prevalence.

\section{Statistical analysis}

Data were presented as numbers and proportions. The random effect model was used for combining the prevalence of sarcopenia. The sex-specific prevalence of sarcopenia and 95\% confidence interval $(\mathrm{CI})$ were calculated 
using the Binomial Exact Method. Heterogeneity was assessed by subgroup analysis. The heterogeneity of studies greater than $50 \%$ indicated severe heterogeneity. The publication bias was assessed by Begg's test. Given the fact that prevalence does not have a normal distribution, a prevalence index was first calculated using the logit of prevalence and the standard error of logit prevalence, and then tests were done. All meta-analysis methods were performed using STATA (Release 12. statistical software. College Station, Texas: STATA Corp LP).

\section{Results}

The literature searches yielded 2329 articles (including 559 duplicates). According to inclusion criteria, we assessed titles and abstracts and 115 articles were selected. After reviewing the full texts, thirty five studies were eligible for the meta- analysis (Fig. 1).

A total of 58404 individuals, $32642(55.9 \%)$ men and 25762 (44.1\%) women; respectively, from the general population were examined through these studies. Twenty one studies were distributed in Asia and there were also 14 studies from non-Asian countries. A total of 18 studies used the Dual Energy X-Ray Absorptiometry (DXA) to assess muscle mass and 16 studies used the Bio-electrical Impedance Analysis (BIA). In a study were used both methods to assess of muscle mass [13]. Characteristics of studies included in the analyses are described in Table 1.

\section{Meta- analysis}

To explore the sources of heterogeneity, eight subgroups were analyzed. These groups were gender, region of the study, and method of muscle mass measurement. Then, we assessed region and method in each gender, separately. The overall estimates of prevalence was 10\% (95\% CI: $8-12 \%)$ in men and $10 \%$ (95\% CI: $813 \%)$ in women, respectively. Substantial heterogeneity observed in men $(\mathrm{df}=46, \mathrm{I} 2=98.11 \%, P=0.00)$ and women $(\mathrm{df}=$ 45 , I2 $=98.07 \%, P=0.00)$. Because the cut-off points of sarcopenia parameters are different in men and women, the prevalence of this disease has been reported in both genders separately. Individuals in non-Asian countries were more likely to be sarcopenic than Asian countries among both genders (11\% vs $10 \%$ in men, $13 \%$ vs $9 \%$ in women) (Data not shown).

As shown in Fig. 2, BIA had influence on the prevalence of sarcopenia in men and women, respectively [13\% (7-19\%) in men; 13\% (9-19\%) in women]. When the method DXA was used to measure muscle mass, the prevalence of sarcopenia was lower in both genders [8\% (7-9\%) in men; 8\% (6-11\%) in women] (Fig. 3).

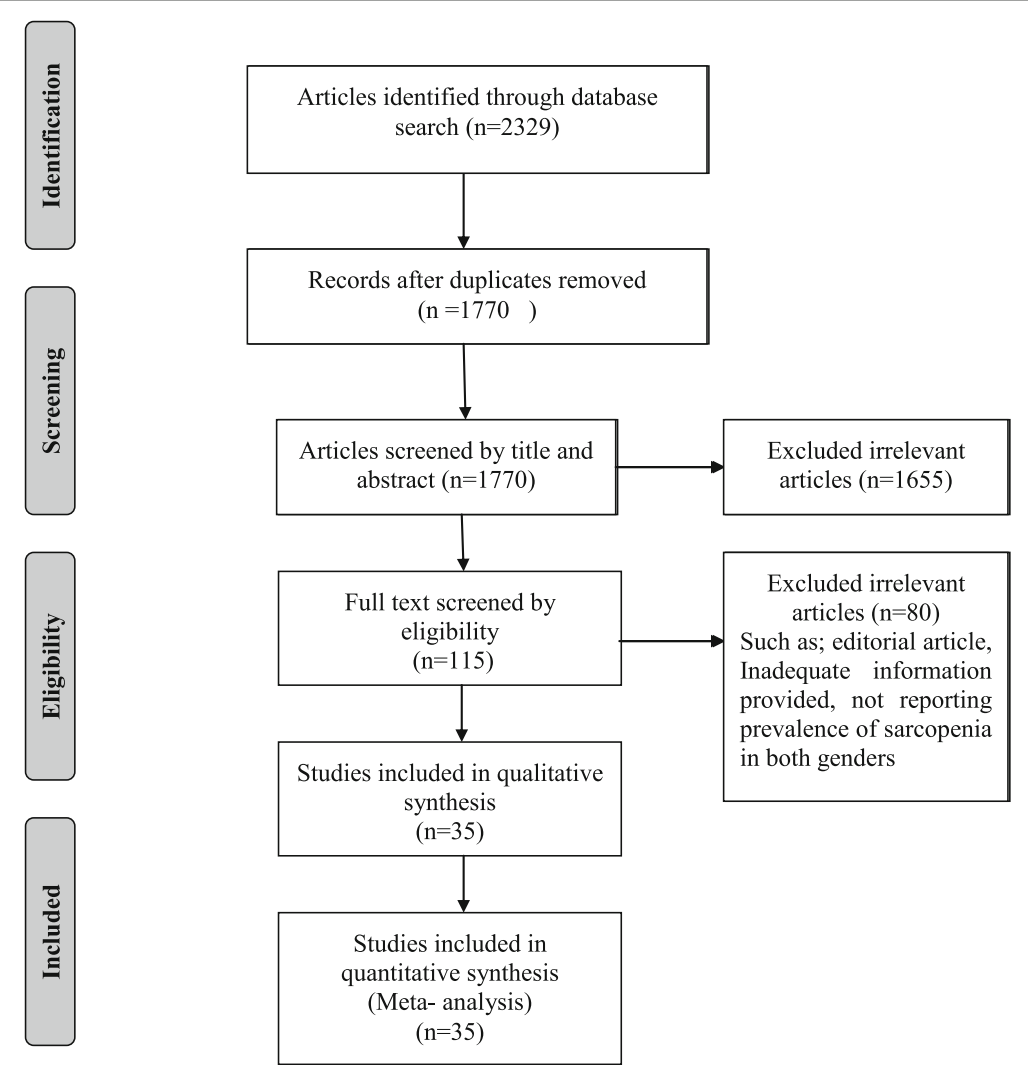

Fig. 1 Flow diagram of literature search 
Table 1 Characteristics of studies included in the sarcopenia prevalence meta-analysis in healthy ageing adults

\begin{tabular}{|c|c|c|c|c|c|c|}
\hline \multirow[t]{2}{*}{ Studies } & \multirow{2}{*}{$\begin{array}{l}\text { Region } \\
\text { (Asia/ } \\
\text { Non } \\
\text { Asia) }\end{array}$} & \multirow{2}{*}{$\begin{array}{l}\text { Sample (N) } \\
\text { Total-Male- } \\
\text { Female }\end{array}$} & \multirow{2}{*}{$\begin{array}{l}\text { Assessment } \\
\text { method for } \\
\text { muscle } \\
\text { mass }\end{array}$} & \multicolumn{3}{|l|}{ Prevalence } \\
\hline & & & & $\begin{array}{l}\text { Total } \\
N(\%)\end{array}$ & $\begin{array}{l}\text { Male } \\
\text { N (\%) }\end{array}$ & $\begin{array}{l}\text { Female } \\
N(\%)\end{array}$ \\
\hline Htun N.C, et al. 2016 [27] & Asia & 1921-976-945 & DXA & $248(13.3)$ & $101(10.34)$ & $147(16.56)$ \\
\hline Borg S.T, et al. 2016 [28] & Non Asia & $227-110-117$ & $\mathrm{BIA}$ & $53(23.0)$ & $25(22.73)$ & $28(23.93)$ \\
\hline Brown J. C, et al. 2016 [29] & Non Asia & $4425-1925-2500$ & $\mathrm{BIA}$ & $1618(36.0)$ & $862(44.8)$ & $756(30.24$ \\
\hline Chan R, et al. 2016 [30] & Asia & 3957-1979-1978 & DXA & $290(7.30)$ & $185(9.30)$ & $105(5.30)$ \\
\hline Jung H. W, et al. 2016 [31] & Asia & $382-167-215$ & $\mathrm{BIA}$ & $105(27.80)$ & $46(28.10)$ & $59(27.44)$ \\
\hline Han D. S, et al. 2016 [32] & Asia & $878-402-476$ & $\mathrm{BIA}$ & $29(3.3)$ & $27(6.7)$ & $2(0.4)$ \\
\hline Spira D, et al. 2016 [33] & Non Asia & $1405-622-783$ & DXA & $58(4.10)$ & $40(6.40)$ & $18(2.30)$ \\
\hline Bianchi L, et al. 2015 [34] & Non Asia & $538-250-288$ & $\mathrm{BIA}$ & $55(10.20)$ & $19(7.60)$ & $36(12.50)$ \\
\hline Han P, et al. 2016 [35] & Asia & $657-216-441$ & DXA & $64(9.70)$ & $21(9.7)$ & $43(9.8)$ \\
\hline Huang C.Y, et al. 2016 [36] & Asia & $731-386-345$ & DXA & $50(6.80)$ & $36(9.30)$ & $14(4.10)$ \\
\hline Siva Neto L.S, et al. 2016 [37] & Non Asia & $70-31-39$ & DXA & $7(10.0)$ & $5(16.10)$ & $2(5.10)$ \\
\hline Han P, et al. 2016 [38] & Asia & $1069-470-606$ & $\mathrm{BIA}$ & $99(9.30)$ & $30(6.40)$ & $69(11.50)$ \\
\hline Velazquez-Alva M.C, et al. 2015 [39] & Non Asia &,,-- 137 & DXA & - & - & $20(14.60)$ \\
\hline Wang Y. J, et al. 2015 [40] & Asia & $316-164-152$ & DXA & $94(29.75)$ & $43(26.20)$ & $51(33.55)$ \\
\hline Pereira F. B, et al. 2015 [41] & Non Asia & $-, 198,-$ & DXA & - & $20(10.10)$ & - \\
\hline Cawthon P.M, et al. 2015 [42] & Non Asia & $-, 5934,-$ & DXA & - & $277(4.70)$ & - \\
\hline Meng N. H, et al. 2015 [43] & Asia & $771-412-359$ & DXA & $44(5.70)$ & $35(8.40)$ & $9(2.60)$ \\
\hline Wen X, et al. 2015 [44] ${ }^{\text {a }}$ & Asia & $286-136-150$ & DXA & & & \\
\hline 1 & & & & $17(5.90)$ & $10(7.40)$ & $7(4.70)$ \\
\hline 2 & & & & $9(3.14)$ & $8(5.90)$ & $1(0.70)$ \\
\hline 3 & & & & $1(0.35)$ & $1(0.80)$ & $0(0.00)$ \\
\hline Bischoff-Ferrari H.A, et al. 2015 [10] ${ }^{a}$ & Non Asia & $445-199-246$ & DXA & & & \\
\hline 1 & & & & $31(7.10)$ & $13(6.60)$ & $18(7.40)$ \\
\hline 2 & & & & $22(5.00)$ & $7(3.60)$ & $15(6.20)$ \\
\hline 3 & & & & $12(2.70)$ & $6(3.10)$ & $6(2.50)$ \\
\hline Nishiguchi S, et al. 2015 [45] & Asia &,,-- 273 & $\mathrm{BIA}$ & - & - & $22(8.06)$ \\
\hline Beaudart C, et al. 2014 [46] ${ }^{a}$ & Non Asia & $400-157-243$ & DXA & & & \\
\hline 1 & & & & $61(15.20)$ & $23(14.65)$ & $38(15.63)$ \\
\hline 2 & & & & $72(18.00)$ & $23(14.65)$ & $49(20.16)$ \\
\hline Yoshida D, et al. 2014 [47] & Asia & $4811-2343-2468$ & $\mathrm{BIA}$ & $360(7.50)$ & $192(8.20)$ & $168(6.80)$ \\
\hline Tanimoto Y, et al. 2014 [48] & Asia & 1110-372-738 & $\mathrm{BIA}$ & $160(14.41)$ & $50(13.40)$ & $110(14.90)$ \\
\hline Wu C.H, et al. 2014 [49] & Asia & $549-285-264$ & $\mathrm{BIA}$ & $39(7.10)$ & $11(3.86)$ & $28(10.61)$ \\
\hline Yu R, et al. 2014 [50] & Asia & $4000-2000-2000$ & DXA & $293(7.32)$ & $187(9.35)$ & $106(5.30)$ \\
\hline Akune T, et al. 2014 [51] & Asia & $1000-349-651$ & $\mathrm{BIA}$ & $129(12.90)$ & $48(13.80)$ & $81(12.40)$ \\
\hline Dam T. T, et al. 2014 [52] ${ }^{a}$ & Non Asia & $10,063-7113-2950$ & DXA & & & \\
\hline 1 & & & & $710(7.0)$ & $362(5.10)$ & $348(11.80)$ \\
\hline 2 & & & & $768(7.63)$ & $376(5.30)$ & $392(13.30)$ \\
\hline Wu I.C.et al. 2014 [11 $]^{a}$ & Asia & 2867-1431-1436 & $\mathrm{BIA}$ & & & \\
\hline 1 & & & & $50(1.74)$ & $33(2.30)$ & $17(1.18)$ \\
\hline 2 & & & & $105(3.70)$ & $55(3.84)$ & $50(3.50)$ \\
\hline
\end{tabular}


Table 1 Characteristics of studies included in the sarcopenia prevalence meta-analysis in healthy ageing adults (Continued)

\begin{tabular}{|c|c|c|c|c|c|c|}
\hline Ishii S, et al. 2014 [53] & Asia & 1971-977-994 & $\mathrm{BIA}$ & $359(18.21)$ & $139(14.20)$ & $220(22.10)$ \\
\hline Pagotto V, et al. 2014 [13] ${ }^{\mathrm{a}}$ & Non Asia & $132-52-81$ & & & & \\
\hline 1 & & & DXA & $17(13.00)$ & $8(15.40)$ & $9(11.40)$ \\
\hline 2 & & & DXA & $22(16.80)$ & $8(15.40)$ & $14(17.28)$ \\
\hline 3 & & & DXA & $48(36.60)$ & $12(23.10)$ & $36(44.44)$ \\
\hline 4 & & & $\mathrm{BIA}$ & $40(30.50)$ & $13(25.00)$ & $27(33.33)$ \\
\hline 5 & & & $\mathrm{BIA}$ & $23(17.60)$ & $8(15.40)$ & $15(19.0)$ \\
\hline Yu S, et al. 2014 [54] ${ }^{a}$ & Non Asia & $986-611-375$ & DXA & & & \\
\hline 1 & & & & $16(1.6)$ & $15(2.5)$ & $1(0.3)$ \\
\hline 2 & & & & $73(7.4)$ & $38(6.2)$ & $35(9.3)$ \\
\hline Volpato S, et al. 2014 [55] & Non Asia & $730-345-385$ & $\mathrm{BIA}$ & $55(7.50)$ & $19(5.51)$ & $36(9.35)$ \\
\hline LeeW.J, et al. 2013 [56] a & Asia & $386-223-163$ & DXA & & & \\
\hline 1 & & & & $30(7.80)$ & $24(10.80)$ & $6(3.70)$ \\
\hline 2 & & & & $16(4.10)$ & $13(5.80)$ & $3(1.80)$ \\
\hline Yamada M, et al. 2013 [57] & Asia & $1882-568-1314$ & $\mathrm{BIA}$ & $414(22.0)$ & $124(21.80)$ & $290(22.10)$ \\
\hline Tanimoto Y. et al. 2012 [58] & Asia & 1158-364-794 & $\mathrm{BIA}$ & $126(10.90)$ & $41(11.30)$ & $85(10.70)$ \\
\hline
\end{tabular}

DXA Dual Energy X-Ray Absorptiometry, BIA Bio-electrical Impedance Analysis

a Consists of different methods or definition for estimation of prevalence of sarcopenia

In the non-Asian subgroup, the prevalence of sarcopenia among men was higher than in the Asian population when the method BIA was used to measure muscle mass (19 vs $10 \%)$. However, in the studies that DXA was used, the prevalence of sarcopenia was 9\% in Asian men and $6 \%$ in non-Asian men (Fig. 4).

Women in non-Asian countries were more likely to be sarcopenic than their counterparts in Asia in both tools for measurement of muscle mass [ 20 vs $11 \%$ with BIA method; 10 vs 6\% with DXA method) (Fig. 5).

According to Begg's test the value for $\mathrm{Z}$ equaled $1.79(p=0.08)$ in men and $0.31(p=0.76)$ in women, that showed no evidence of substantial publication bias.

\section{Discussion}

With increase in the world's older population, sarcopenia is becoming a serious global public health problem. Based on the results of this meta- analysis, despite the high heterogeneity of prevalence estimates across primary studies, the overall prevalence of sarcopenia was $10 \%$. In the non-Asian countries, the prevalence of sarcopenia was more likely than in the Asian individuals. BIA had influence on the prevalence of sarcopenia in

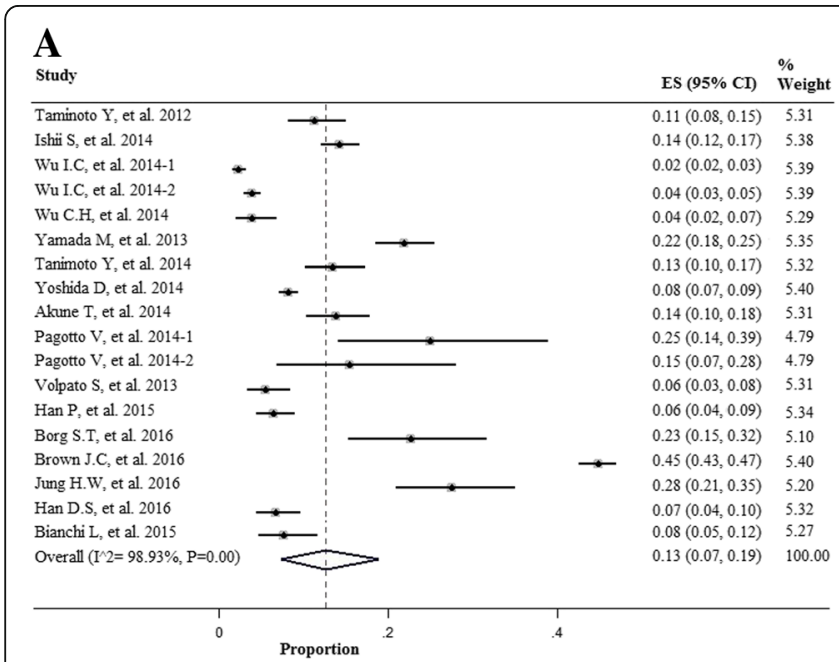

B

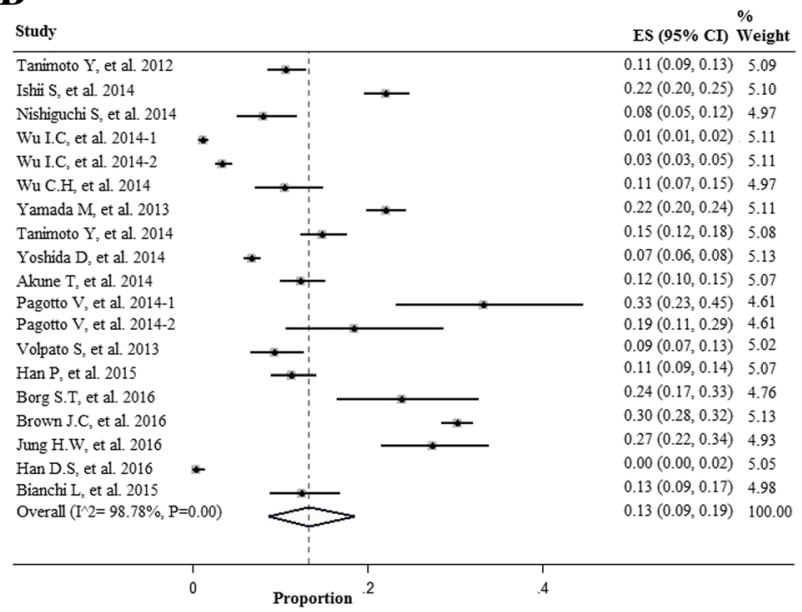

Fig. 2 Forest plot of the studies on sarcopenia prevalence by using the Bio-electrical Impedance Analysis (BIA) in both genders; a Men; b Women 


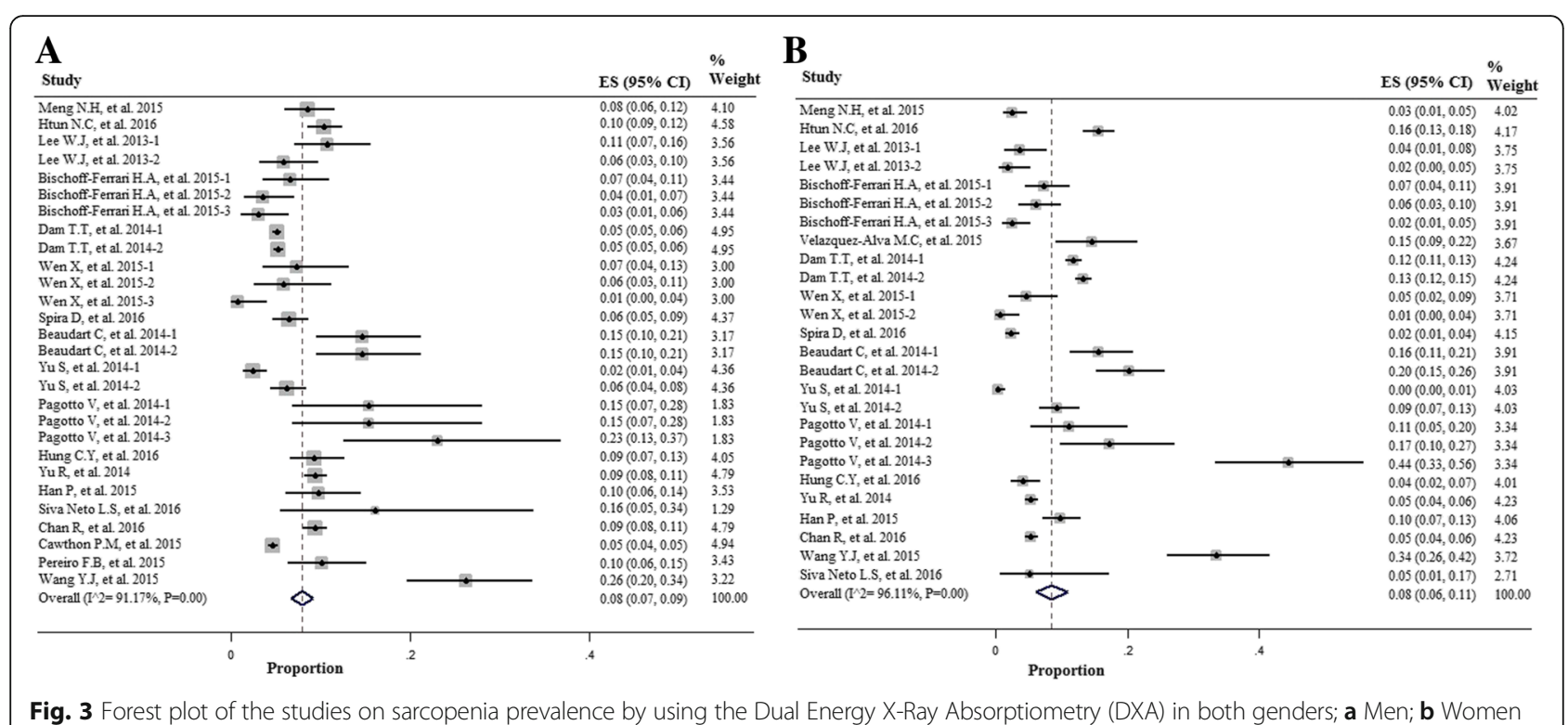

both genders. Considering that sarcopenia has developed a more important issue in elderly, we presumed that an estimate of prevalence in both genders by meta-analysis would be necessary for further strategies of prevention or treatment of sarcopenia.

However, the prevalence of sarcopenia was similar in both genders in this systematic review; the differences have been shown in previous studies. Gender and age differences in body composition are well known. Regarding the association between sex and sarcopenia, results are inconsistent. Some studies $[5,14]$ reported higher relative reduction of muscle mass in men than in women and therefore, the highest prevalence of sarcopenia (50\%) was found among men older than 80 years while only $43.8 \%$ of the women of the same age group corresponded to the definitions of sarcopenia $[1,15,16]$. In contrast, other studies yielded high rates of sarcopenia among women younger than 80 years [16]. Various endogenous and exogenous factors influence on prevalence of sarcopenia. Furthermore hormonal changes which enhance the decrease of muscle mass occur more slowly in men than in women. After menopausal transition, the concentrations of sex steroids, both estrogens and androgens, decrease dramatically [17]. In men, the decline of
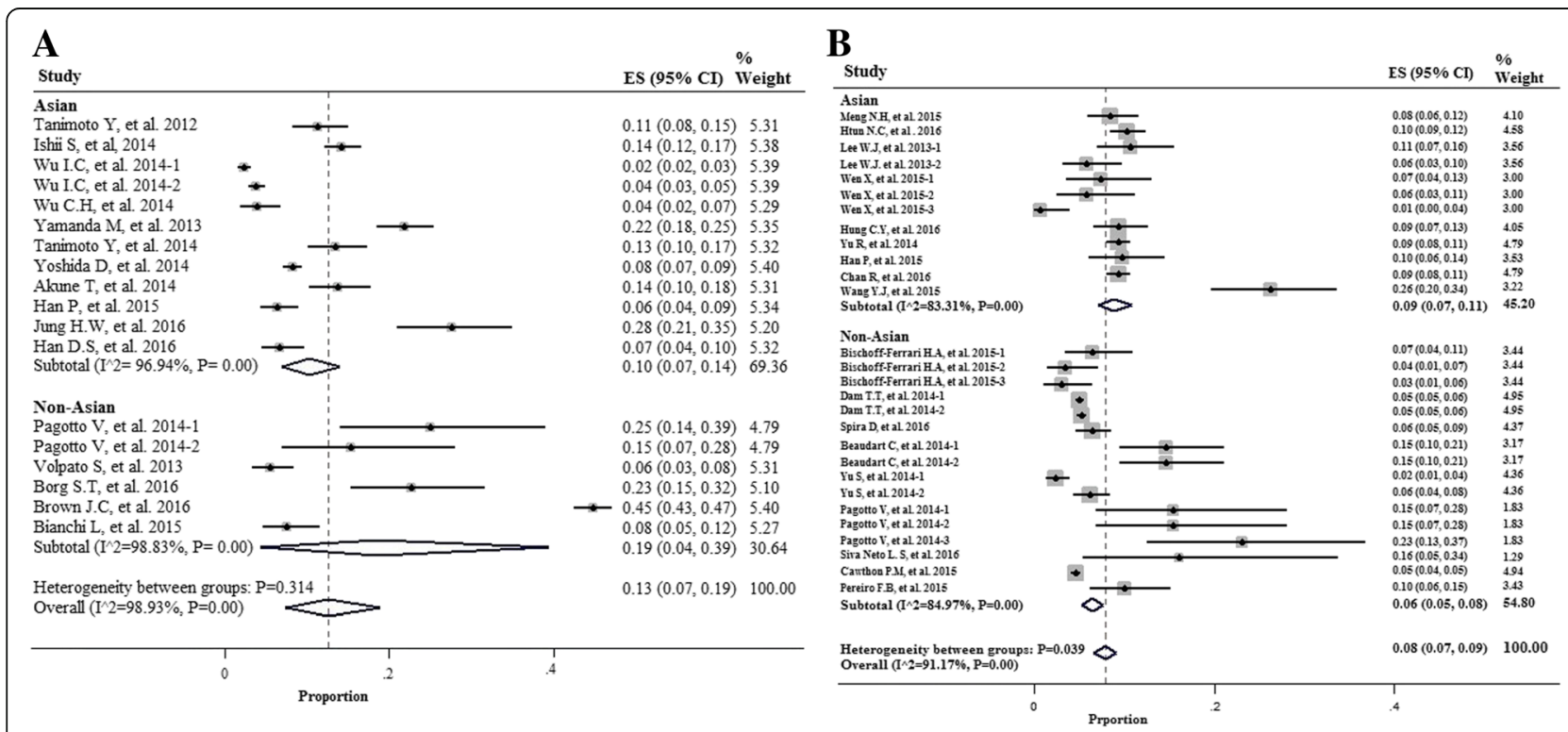

Fig. 4 Forest plot of the studies on sarcopenia prevalence by using the Bio-electrical Impedance Analysis (BIA) a and the Dual Energy X-Ray Absorptiometry (DXA) $\mathbf{b}$ according to region of study (Asia \& Non- Asian) in Men 

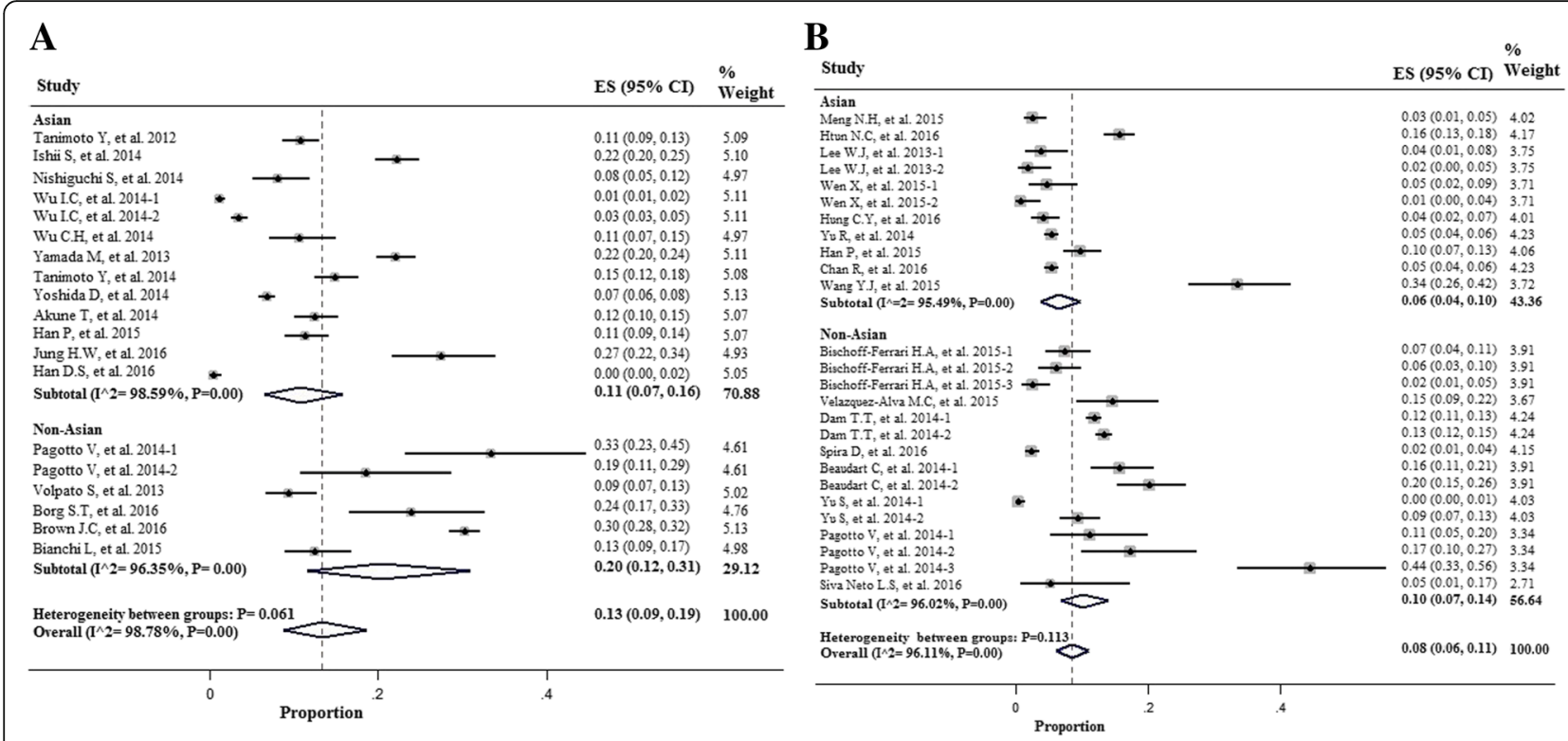

Fig. 5 Forest plot of the studies on sarcopenia prevalence by using the Bio-electrical Impedance Analysis (BIA) a and the Dual Energy X-Ray Absorptiometry (DXA) b according to region of study (Asia \& Non- Asian) in Women

sex steroids is much slower than in women [18]. This advanced decrease of anabolic acting androgens in women may be one important factor in explaining the significantly higher prevalence of sarcopenia among women ageing between 60 and 70 years. After the eighth decade of life, testosterone concentrations in human males decline rapidly which may contribute to the decrease in lean body mass and the increase in sarcopenia.

In present review, we just have sub grouped the studies according to gender, and the studies that had enrolled only participants in the age group above 70 years, were excluded. Also, a pooled analysis of different age groups was not conducted due to inadequate data in each subgroup. Therefore, it seems similar prevalence of sarcopenia in both genders is due to these items.

In relation to the type of elderly population, the prevalence of sarcopenia was higher in elderly people in the rehabilitation units, followed by nursing homes and hospitalized in previous studies [19-21]. Therefore, we excluded these studies that may overestimate its prevalence.

Within our expectation, some variations in estimates of prevalence of sarcopenia were found in sub groups. This variability was not explained by region, and tool of muscle mass measurement. However, some of these variables did change the estimated prevalence of sarcopenia in both genders. The pooled prevalence was higher among non-Asian individuals than in the Asian population in both genders (11 vs $10 \%$ in men, 12 vs $9 \%$ in women).

These results can be attributed to racial characteristics, body size, cultural background, dietary regimes, and life quality of the elderly between the Asian and non-Asian individuals in different countries. Also, the cut-off points for the Asian populations [9] are lower than for the nonAsian individuals [7] in both genders, with young people of the same ethnic group as reference. In addition, the mean appendicular muscle mass of young Asians was about $15 \%$ lower than that of non- Asians even after height adjustments $[1,22]$. Therefore, low muscle mass in young Asians will effect in lower prevalence of sarcopenia in the old people. Furthermore, sarcopenia may be less prevalent in Asians due to differences in lifestyle such as a better dietary aspect and higher levels of activity than the Western populations, which act as protective factors against sarcopenia [23].

Another important factor to estimate prevalence of sarcopenia is tools that use to evaluate muscle mass and diagnose sarcopenia. However, the EWGSOP and other groups do not recommend the use of specific tools to measure muscle mass and other parameters of sarcopenia, but they suggest DXA and BIA to assess muscle mass [7]. The use of different diagnostic tools may lead to different prevalence of sarcopenia and may therefore have important consequences on clinical researches and development of therapeutic strategies. BIA is known to underestimate fat mass and overestimate muscle mass [24]. Previous studies found that the BIA-based prevalence of sarcopenia was higher than the DXA- based approach $[25,26]$. Also, we found important differences of measured prevalence of sarcopenia whether BIA or DXA. When the BIA was used, the pooled prevalence in both genders was observed to be $13 \%$ in men and women. This positive correlation implies an even higher 
prevalence of sarcopenia among the region. These results suggest that the prevalence of sarcopenia should be tool-based approach dependent.

The limitations of the present review need to be considered. First, not all studies presented data according to age group; therefore, we could not estimate the prevalence for each age subgroup. Second, grouping according to different cut-off points for parameters of sarcopenia and different definitions were led to reader's confusion. So, analyses were based on sex, region and tool of muscle mass measurement. Third, characteristics of residents (e.g. rural or urban) could not be assessed due to insufficient data from the majority of publications.

One of the strength of the present systematic review and meta- analysis was the first study that only included studies that were conducted in the general population. Other strength of this study was that only high quality articles were included. Most of the excluded articles either had a low quality based on STROBE quality assessment forms and therefore did not present reliable data, or lacked information required for the review, such as adequate descriptive information about the population of their study, or a separate sarcopenia prevalence in different sex, which can make it difficult to use in a meta-analysis.

\section{Conclusion}

Despite the differences encountered between the studies, regarding diagnostic tools used to measure of muscle mass and different regions of the world for estimating parameters of sarcopenia, present systematic review revealed that a substantial proportion of the old people has sarcopenia, even in healthy populations. However, sarcopenia is as a consequence of the aging progress, early diagnosis of those at risk can prevent some adverse outcomes. Therefore, future researches are needed to find a consensus regarding the tools that must be used in the context of diagnosing and screening of sarcopenia. Therefore, it is necessary that the simple and valid methods for diagnosing and screening of sarcopenia used in clinical practice.

\section{Abbreviations}

AWGS: Asian Working Group for Sarcopenia; BIA: Bio-electrical impedance analysis; DXA: Dual Energy X-Ray Absorptiometry; EWGSOP: European Working Group on Sarcopenia in Older People; IWGS: International Working Group on Sarcopenia; STROBE: Strengthening the Reporting of Observational studies in Epidemiology

\section{Acknowledgment}

Not applicable.

Funding

This study was not supported by any fund.

Availability of data and materials

Data supporting the results were reported in the manuscript.
Authors' contribution

GS, AS: writing first draft of manuscript. RH, BL: concept and study design. GS, ZA: data collection and critical review, AAK: Analysis and/or Interpretation. All authors read and approved the final manuscript.

\section{Competing interest}

The authors declare that they have no competing interest.

Consent for publication

Not applicable.

Ethics approval and consent to participate

Not applicable.

\section{Publisher's Note}

Springer Nature remains neutral with regard to jurisdictional claims in published maps and institutional affiliations.

\section{Author details}

${ }^{1}$ Chronic Diseases Research Center, Endocrinology and Metabolism Population Sciences Institute, Tehran University of Medical Sciences, Tehran, Iran. ${ }^{2}$ Department of Health Sciences Education Development, School of Public Health, Tehran University of Medical Sciences, Tehran, Iran.

${ }^{3}$ Endocrinology and Metabolism Research Center, Endocrinology and Metabolism Clinical Sciences Institute, Tehran University of Medical Sciences, Tehran, Iran. ${ }^{4}$ Endocrinology and Metabolism Research Center (EMRC), Dr Shariati Hospital, North Karegar St, Tehran 14114, Iran.

Received: 8 February 2017 Accepted: 9 May 2017

Published online: 16 May 2017

\section{References}

1. Baumgartner RN, Koehler KM, Gallagher D, Romero L, Heymsfield SB, Ross RR, et al. Epidemiology of sarcopenia among the elderly in New Mexico. Am J Epidemiol. 1998;147(8):755-63. Epub 1998/04/29.

2. Lauretani F, Russo CR, Bandinelli S, Bartali B, Cavazzini C, Di lorio A, et al. Age-associated changes in skeletal muscles and their effect on mobility: an operational diagnosis of sarcopenia. J Appl Physiol. 2003;95(5):1851-60. Epub 2003/10/14

3. Rizzoli R, Reginster JY, Arnal JF, Bautmans I, Beaudart C, Bischoff-Ferrari H, et al. Quality of life in sarcopenia and frailty. Calcif Tissue Int. 2013;93(2):101-20. Epub 2013/07/06.

4. Rantanen T. Muscle strength, disability and mortality. Scand J Med Sci Sports. 2003;13(1):3-8. Epub 2003/01/22.

5. Gallagher D, Visser M, De Meersman RE, Sepulveda D, Baumgartner RN, Pierson RN, et al. Appendicular skeletal muscle mass: effects of age, gender, and ethnicity. J Appl Physiol. 1997;83(1):229-39. Epub 1997/07/01.

6. Pagotto V, Silveira EA. Methods, diagnostic criteria, cutoff points, and prevalence of sarcopenia among older people. Scientific World Journal. 2014;2014:231312. Epub 2015/01/13.

7. Cruz-Jentoft AJ, Baeyens JP, Bauer JM, Boirie Y, Cederholm T, Landi F, et al. Sarcopenia: European consensus on definition and diagnosis: report of the European working group on sarcopenia in older people. Age Ageing. 2010; 39(4):412-23. Epub 2010/04/16.

8. Fielding RA, Vellas B, Evans WJ, Bhasin S, Morley JE, Newman AB, et al. Sarcopenia: an undiagnosed condition in older adults. Current consensus definition: prevalence, etiology, and consequences. International working group on sarcopenia. J Am Med Dir Assoc. 2011;12(4):249-56. Epub 2011/04/30.

9. Chen LK, Liu LK, Woo J, Assantachai P, Auyeung TW, Bahyah KS, et al. Sarcopenia in Asia: consensus report of the Asian working group for sarcopenia. J Am Med Dir Assoc. 2014;15(2):95-101. Epub 2014/01/28.

10. Bischoff-Ferrari HA, Orav JE, Kanis JA, Rizzoli R, Schlogl M, Staehelin HB, et al. Comparative performance of current definitions of sarcopenia against the prospective incidence of falls among community-dwelling seniors age 65 and older. Osteoporos Int. 2015;26(12):2793-802. Epub 2015/06/13.

11. Wu IC, Lin CC, Hsiung CA, Wang CY, Wu CH, Chan DC, et al. Epidemiology of sarcopenia among community-dwelling older adults in Taiwan: a pooled analysis for a broader adoption of sarcopenia assessments. Geriatr Gerontol Int. 2014;14 Suppl 1:52-60. Epub 2014/01/24. 
12. Vandenbroucke JP, Von Elm E, Altman DG, Gotzsche PC, Mulrow CD, Pocock SJ, et al. [Strengthening the reporting of observational studies in epidemiology (STROBE): explanation and elaboration]. Gac Sanit. 2009; 23(2):158. Epub 2009/03/03. Mejorar la comunicacion de estudios observacionales en epidemiologia (STROBE): explicacion y elaboracion.

13. Pagotto V, Silveira EA. Applicability and agreement of different diagnostic criteria for sarcopenia estimation in the elderly. Arch Gerontol Geriatr. 2014; 59(2):288-94. Epub 2014/06/18.

14. Forbes GB. Longitudinal changes in adult fat-free mass: influence of body weight. Am J Clin Nutr. 1999;70(6):1025-31. Epub 1999/12/03.

15. lannuzzi-Sucich M, Prestwood KM, Kenny AM. Prevalence of sarcopenia and predictors of skeletal muscle mass in healthy, older men and women. J Gerontol A Biol Sci Med Sci. 2002;57(12):M772-7. Epub 2002/11/29.

16. Kirchengast $S$, Huber J. Gender and age differences in lean soft tissue mass and sarcopenia among healthy elderly. Anthropol Anz. 2009;67(2):139-51. Epub 2009/09/11.

17. Burger HG, Dudley EC, Robertson DM, Dennerstein L. Hormonal changes in the menopause transition. Recent Prog Horm Res. 2002;57:257-75. Epub 2002/05/23.

18. Juul A, Skakkebaek NE. Androgens and the ageing male. Hum Reprod Update. 2002;8(5):423-33. Epub 2002/10/26

19. Rubio-Maicas C, Duarte-Alfonso E, Beseler-Soto MR, Moreno-Munoz I, MoralMoral P, Merino-Torres JF. Prevalence of sarcopenia in a media and long stay Unit. Rev Clin Esp. 2014;214(6):303-8. Epub 2014/05/07. Prevalencia de sarcopenia en una unidad de media y larga estancia.

20. Landi F, Liperoti R, Fusco D, Mastropaolo S, Quattrociocchi D, Proia A, et al Sarcopenia and mortality among older nursing home residents. J Am Med Dir Assoc. 2012;13(2):121-6. Epub 2011/08/23.

21. Smoliner C, Sieber CC, Wirth R. Prevalence of sarcopenia in geriatric hospitalized patients. J Am Med Dir Assoc. 2014;15(4):267-72. Epub 2014/04/01.

22. Lau EM, Lynn HS, Woo JW, Kwok TC, Melton 3rd LJ. Prevalence of and risk factors for sarcopenia in elderly Chinese men and women. J Gerontol A Biol Sci Med Sci. 2005;60(2):213-6. Epub 2005/04/09.

23. Woo J, Leung J, Sham A, Kwok T. Defining sarcopenia in terms of risk of physical limitations: a 5-year follow-up study of 3,153 chinese men and women. J Am Geriatr Soc. 2009:57(12):2224-31. Epub 2009/11/21.

24. Janssen I, Heymsfield SB, Baumgartner RN, Ross R. Estimation of skeletal muscle mass by bioelectrical impedance analysis. J Appl Physiol. 2000;89(2): 465-71. Epub 2000/08/05.

25. Chien MY, Huang TY, Wu YT. Prevalence of sarcopenia estimated using a bioelectrical impedance analysis prediction equation in communitydwelling elderly people in Taiwan. J Am Geriatr Soc. 2008;56(9):1710-5. Epub 2008/08/12.

26. Lee JS, Auyeung TW, Kwok T, Lau EM, Leung PC, Woo J. Associated factors and health impact of sarcopenia in older chinese men and women: a crosssectional study. Gerontology. 2007;53(6):404-10. Epub 2007/08/19.

27. Htun NC, Ishikawa-Takata K, Kuroda A, Tanaka T, Kikutani T, Obuchi SP, et al. Screening for malnutrition in community dwelling older Japanese: preliminary development and evaluation of the Japanese nutritional risk screening too (NRST). J Nutr Health Aging. 2016;20(2):114-20. Epub 2016/01/27.

28. Ter Borg S, de Groot LC, Mijnarends DM, de Vries JH, Verlaan S, Meijboom S, et al. Differences in nutrient intake and biochemical nutrient status between sarcopenic and nonsarcopenic older adults-results from the Maastricht sarcopenia study. J Am Med Dir Assoc. 2016;17(5):393-401. Epub 2016/01/31

29. Brown JC, Harhay MO, Harhay MN. Sarcopenia and mortality among a population-based sample of community-dwelling older adults. J Cachexia Sarcop enia Muscle. 2016;7(3):290-8. Epub 2016/05/31.

30. Chan R, Leung J, Woo J. A prospective cohort study to examine the association between dietary patterns and sarcopenia in Chinese community-dwelling older people in Hong Kong. J Am Med Dir Assoc. 2016;17(4):336-42. Epub 2016/01/18.

31. Jung HW, Jang IY, Lee $Y S$, Lee CK, Cho El, Kang WY, et al. Prevalence of frailty and aging-related health conditions in older Koreans in rural communities: a cross-sectional analysis of the aging study of pyeongchang rural area. J Korean Med Sci. 2016:31(3):345-52. Epub 2016/03/10.

32. Han DS, Chang KV, Li CM, Lin YH, Kao TW, et al. Skeletal muscle mass adjusted by height correlated better with muscular functions than that adjusted by body weight in defining sarcopenia. Sci Rep. 2016;6:19457.

33. Spira D, Norman K, Nikolov J, Demuth I, Steinhagen-Thiessen E, Eckardt R. Prevalence and definition of sarcopenia in community dwelling older people. Data from the Berlin aging study II (BASE-II). Z Gerontol Geriatr. 2016;49(2):94-9. Epub 2015/04/17.

34. Bianchi L, Ferrucci L, Cherubini A, Maggio M, Bandinelli S, Savino E, et al. The predictive value of the EWGSOP definition of sarcopenia: results from the InCHIANTI study. J Gerontol A Biol Sci Med Sci. 2016;71(2):259-64. Epub 2015/09/04.

35. Han P, Zhao J, Guo Q, Wang J, Zhang W, Shen S, et al. Incidence, risk factors, and the protective effect of high body mass index against sarcopenia in suburb-dwelling elderly Chinese populations. J Nutr Health Aging. 2016;20(10):1056-60. Epub 2016/12/08.

36. Huang CY, Hwang AC, Liu LK, Lee WJ, Chen LY, Peng LN, et al. Association of dynapenia, sarcopenia, and cognitive impairment among communitydwelling older Taiwanese. Rejuvenation Res. 2016;19(1):71-8. Epub 2015/07/15.

37. Silva Neto LS, Karnikowski MG, Osorio NB, Pereira LC, Mendes MB, Galato D, et al. Association between sarcopenia and quality of life in quilombola elderly in Brazil. Int J Gen Med. 2016;9:89-97. Epub 2016/05/05.

38. Han P, Kang L, Guo Q, Wang J, Zhang W, Shen S, et al. Prevalence and factors associated with sarcopenia in suburb-dwelling older Chinese using the Asian working group for sarcopenia definition. J Gerontol A Biol Sci Med Sci. 2016;71(4):529-35. Epub 2015/08/20.

39. Velazquez-Alva MC, Irigoyen Camacho ME, Lazarevich I, Delgadillo Velazquez J, Acosta Dominguez P, Zepeda Zepeda MA. Comparison of the prevalence of sarcopenia using skeletal muscle mass index and calf circumference applying the European consensus definition in elderly Mexican women. Geriatr Gerontol Int. 2017;17(1):161-70. Epub 2015/11/05.

40. Wang YJ, Wang Y, Zhan JK, Tang ZY, He JY, Tan P, et al. Sarco-osteoporosis: prevalence and association with frailty in Chinese community-dwelling older adults. Int J Endocrinol. 2015;2015:482940. Epub 2015/08/15.

41. Pereira FB, Leite AF, de Paula AP. Relationship between pre-sarcopenia, sarcopenia and bone mineral density in elderly men. Arch Endocrinol Metab. 2015;59(1):59-65. Epub 2015/05/01.

42. Cawthon PM, Blackwell TL, Cauley J, Kado DM, Barrett-Connor E, Lee CG, et al. Evaluation of the usefulness of consensus definitions of sarcopenia in older Men: results from the observational osteoporotic fractures in Men cohort study. J Am Geriatr Soc. 2015;63(11):2247-59. Epub 2015/10/28.

43. Meng NH, Li Cl, Liu CS, Lin CH, Lin WY, Chang CK, et al. Comparison of height- and weight-adjusted sarcopenia in a Taiwanese metropolitan older population. Geriatr Gerontol Int. 2015;15(1):45-53. Epub 2014/01/09.

44. Wen $X, A n P$, Chen WC, Lv Y, Fu Q. Comparisons of sarcopenia prevalence based on different diagnostic criteria in Chinese older adults. J Nutr Health Aging. 2015;19(3):342-7. Epub 2015/03/04.

45. Nishiguchi S, Yamada M, Fukutani N, Adachi D, Tashiro Y, Hotta T, et al. Differential association of frailty with cognitive decline and sarcopenia in community-dwelling older adults. J Am Med Dir Assoc. 2015;16(2):120-4. Epub 2014/09/24

46. Beaudart C, Reginster JY, Slomian J, Buckinx F, Locquet M, Bruyere O. Prevalence of sarcopenia: the impact of different diagnostic cut-off limits. J Musculoskelet Neuronal Interact. 2014;14(4):425-31. Epub 2014/12/20.

47. Yoshida D, Suzuki T, Shimada H, Park H, Makizako H, Doi T, et al. Using two different algorithms to determine the prevalence of sarcopenia. Geriatr Gerontol Int. 2014;14 Suppl 1:46-51. Epub 2014/01/24.

48. Tanimoto $Y$, Watanabe M, Sun W, Sugiura Y, Hayashida I, Kusabiraki T, et al. Sarcopenia and falls in community-dwelling elderly subjects in Japan: defining sarcopenia according to criteria of the European working group on sarcopenia in older people. Arch Gerontol Geriatr. 2014;59(2):295-9. Epub 2014/05/24.

49. Wu CH, Chen KT, Hou MT, Chang YF, Chang CS, Liu PY, et al. Prevalence and associated factors of sarcopenia and severe sarcopenia in older Taiwanese living in rural community: the tianliao Old people study 04 Geriatr Gerontol Int. 2014;14 Suppl 1:69-75. Epub 2014/01/24.

50. Yu R, Leung J, Woo J. Incremental predictive value of sarcopenia for incident fracture in an elderly Chinese cohort: results from the osteoporotic fractures in Men (MrOs) study. J Am Med Dir Assoc. 2014;15(8):551-8. Epub 2014/04/08

51. Akune T, Muraki S, Oka H, Tanaka S, Kawaguchi H, Nakamura K, et al. Exercise habits during middle age are associated with lower prevalence of sarcopenia: the ROAD study. Osteoporos Int. 2014;25(3):1081-8. Epub 2013/10/23

52. Dam TT, Peters KW, Fragala M, Cawthon PM, Harris TB, McLean R, et al. An evidence-based comparison of operational criteria for the presence of sarcopenia. J Gerontol A Biol Sci Med Sci. 2014;69(5):584-90. Epub 2014/04/17. 
53. Ishii S, Tanaka T, Shibasaki K, Ouchi Y, Kikutani T, Higashiguchi T, et al. Development of a simple screening test for sarcopenia in older adults. Geriatr Gerontol Int. 2014;14 Suppl 1:93-101. Epub 2014/01/24.

54. Yu S, Appleton S, Adams R, Chapman I, Wittert G, Visvanathan T, et al. The impact of low muscle mass definition on the prevalence of sarcopenia in older Australians. Biomed Res Int. 2014;2014:361790. Epub 2014/08/29.

55. Volpato S, Bianchi L, Cherubini A, Landi F, Maggio M, Savino E, et al. Prevalence and clinical correlates of sarcopenia in community-dwelling older people: application of the EWGSOP definition and diagnostic algorithm. J Gerontol A Biol Sci Med Sci. 2014;69(4):438-46. Epub 2013/10/03.

56. Lee WJ, Liu LK, Peng LN, Lin MH, Chen LK. Comparisons of sarcopenia defined by IWGS and EWGSOP criteria among older people: results from the I-Lan longitudinal aging study. J Am Med Dir Assoc. 2013;14(7):528.e1-7. Epub 2013/05/15.

57. Yamada M, Nishiguchi S, Fukutani N, Tanigawa T, Yukutake T, Kayama H, et al. Prevalence of sarcopenia in community-dwelling Japanese older adults. J Am Med Dir Assoc. 2013;14(12):911-5. Epub 2013/10/08.

58. Tanimoto $Y$, Watanabe M, Sun W, Sugiura Y, Tsuda Y, Kimura M, et al. Association between sarcopenia and higher-level functional capacity in daily living in community-dwelling elderly subjects in Japan. Arch Gerontol Geriatr. 2012;55(2):e9-13. Epub 2012/07/17.

\section{Submit your next manuscript to BioMed Central and we will help you at every step:}

- We accept pre-submission inquiries

- Our selector tool helps you to find the most relevant journal

- We provide round the clock customer support

- Convenient online submission

- Thorough peer review

- Inclusion in PubMed and all major indexing services

- Maximum visibility for your research

Submit your manuscript at www.biomedcentral.com/submit 